\title{
Effect of Spp1 on nerve degeneration and regeneration after rat sciatic nerve injury
}

\author{
Xingyu Liu ${ }^{1 \dagger}$, Yuhua Sun ${ }^{2 \dagger}$, Huaiqin $\mathrm{Li}^{2}$, Yuting $\mathrm{Li}^{2}$, Meiyuan $\mathrm{Li}^{2}$, Ying Yuan ${ }^{2,3}$, Shusen Cui ${ }^{{ }^{*}}$ and Dengbing Yao ${ }^{2^{*}}$
}

\begin{abstract}
Background: Wallerian degeneration (WD) in injured peripheral nerves is associated with a large number of up- or down-regulated genes, but the effects of these changes are poorly understood. In our previous studies, we reported some key factors that are differentially expressed to activate nerve degeneration and regeneration during WD. Here, we determined the effects of secreted phosphoprotein 1 (Spp1) on WD after rat sciatic nerve injury.

Results: Spp 1 was upregulated from $6 \mathrm{~h}$ to 14 days after sciatic nerve injury. Altered expression of Spp 1 in Schwann cells (SC) resulted in altered mRNA and protein expression levels for cytokines, c-Fos, PKCa and phospho-ERK/ERK and affected SC apoptosis in vitro. Silencing of Spp1 expression in SCs using siRNA technology reduced proliferation and promoted migration of SCs in vitro. By contrast, overexpression of Spp1 promoted proliferation and reduced migration in SCs in vitro. Differential expression of Spp1 after sciatic nerve injury in vivo altered the expression of cytokines, c-Fos, PKCa, and the p-ERK/ERK pathway.

Conclusions: Spp1 is a key regulatory factor that affects nerve degeneration and regeneration through c-Fos, PKCa and $\mathrm{p}$-ERK/ERK pathways after rat sciatic nerve injury. These results shed new light on the role of Spp 1 in nerve degeneration and regeneration during WD.
\end{abstract}

Keywords: Wallerian degeneration, Secreted phosphoprotein 1 (Spp1), Schwann cells, Nerve regeneration, Rat, Sciatic nerve injury

\section{Background}

Wallerian degeneration (WD), the degeneration of the axon distal to a site of transaction, occurs in both axons and myelin after injury to the peripheral nervous system (PNS) [1-3]. The PNS, unlike the central nervous system (CNS), is capable of regeneration after an injury that causes WD processes to begin [4,5]. A number of studies have found that nerve injury not only plays a key role in modulating the activities of Schwann cells but also promotes axonal regeneration by releasing a large number of regeneration-related factors, including cytokines, growth factors, and chemokines [5-7]. Therefore, it is

\footnotetext{
*Correspondence: sscui916@126.com; yaodb@ntu.edu.cn

${ }^{+}$Xingyu Liu and Yuhua Sun contributed equally to this work

${ }^{1}$ China-Japan Union Hospital of Jilin University, 126 Xiantai Road,

Changchun 130033, Jilin, People's Republic of China

${ }^{2}$ School of Life Sciences, Jiangsu Key Laboratory of Neuroregeneration,

Co-Innovation Center of Neuroregeneration, Nantong University, 19 Qixiu

Road, Nnatong 226001, Jiangsu, People's Republic of China

Full list of author information is available at the end of the article
}

important to elucidate the key factors involved in regulating the degeneration and regeneration that occurs in the PNS after injury [8-11]. The molecular mechanisms regulating WD are not yet completely understood, but understanding the factors that regulate rapid responses during WD may reveal the mechanisms underpinning nerve repair and regeneration [12-16].

Secreted phosphoprotein 1 (Spp1) belongs to the family of secreted acidic proteins. It has a large number of consensus sequence sites and multiple phosphorylation sites and binds to several integrin receptors, which have been well established to function in cell adhesion, migration, and survival [17]. Spp1 is expressed in a range of immune cells and reported to act as an immune modulator, which promotes cell recruitment to inflammatory sites [17]. It also functions as an adhesion protein involved in cell attachment and wound healing [17, 18]. Stimulation of Spp1 expression leads to an increase in cell pro-inflammatory cytokine levels, although the regulatory pathways are not yet known [17-19]. 
We previously reported on gene expression signal flow and pathways regulated by key factors as determined by microarray analyses, such as claudins, transforming growth factor beta 1, Spp1, and toll-like receptor 4, during the processes of WD after rat sciatic nerve injury [1115]. Here, we examined the effect of Spp1 on cytokine release, cell apoptosis, cell migration and proliferation, and signaling pathways in vitro and in vivo.

\section{Methods}

\section{Animal model}

Male Sprague-Dawley rats (180-200 g) were provided by the Experimental Animal Center of Nantong University. The rats were randomly divided into eight groups (six rats per group) and underwent sciatic neurectomy. All animal tests were conducted in accordance with the US National Institutes of Health's Guide for the Care and Use of Laboratory Animals and by the Key Laboratory of Neuroregeneration Guidelines for the Care and Use of Laboratory Animals. The Institutional Animal Care and Use Committee of Nantong University approved all protocols used in this study.

The rats were anesthetized using an injection of complex narcotics $(85 \mathrm{mg} / \mathrm{kg}$ trichloroacetaldehyde monohydrate, $42 \mathrm{mg} / \mathrm{kg}$ magnesium sulfate, and $17 \mathrm{mg} / \mathrm{kg}$ sodium pentobarbital), and the sciatic nerve was identified and lifted through an incision on the lateral aspect of the mid-thigh of the right hind limb. The sciatic nerve was cut, and a 1-cm segment was excised. One group of rats was immediately used in experiments $(0 \mathrm{~h})$, and the other groups were used 6,12 , and $24 \mathrm{~h}$ as well as $1,2,3$, and 4 weeks after the surgery. The 0 -h animals received sham operations.

\section{Primary culture of Schwann cells}

The rats for this experiment were provided by the Experimental Animal Center of Nantong University. The rats were sacrificed, and Schwann cells (SCs) were isolated from the sciatic nerves. The SCs were treated with antiThy1.1 antibody (Sigma, St Louis, MO) and rabbit complement (Invitrogen, Carlsbad, CA) to remove fibroblasts as previously described [20]. SCs were cultured from the sciatic nerves of 1-day-old Sprague-Dawley rats as previously described [20]. Primary cultures of Schwann cells were maintained in Dulbecco's modified Eagle's medium (DMEM) supplemented with $10 \%$ fetal bovine serum at $37^{\circ} \mathrm{C}$ in a humidified $5 \% \mathrm{CO}_{2}$ atmosphere.

\section{Spp1 siRNA transfection of Schwann cells}

Three different small interfering RNAs (siRNAs) (Table 1) were used to perform RNA interference. SCs were transfected with Spp1 siRNAs (Integrated Biotech Solutions, Shanghai, China) using Lipofectamine
Table 1 Spp1 siRNA primers

\begin{tabular}{|c|c|}
\hline Gene & Sequence \\
\hline NC & $\begin{array}{l}\text { F: 5' UUCUCCGAACGUGUCACGUTT 3' } \\
\text { R: 5' ACGUGACACGUUCGGAGAATT 3' }\end{array}$ \\
\hline Spp1-1 & $\begin{array}{l}\text { F: } 5^{\prime} \text { CUAGAUGUCGGUGUCCCUUGC } 3^{\prime} \\
\text { R: 5' UGAAUGUUGCUGCGCAUCAUG 3' }\end{array}$ \\
\hline Spp1-2 & $\begin{array}{l}\text { F: 5' CGAUCGAUAGUGCCGAGAAGC } 3^{\prime} \\
\text { R: 5' UUCUCGGCACUAUCGAUCGCA } 3^{\prime}\end{array}$ \\
\hline Spp1-3 & $\begin{array}{l}\text { F: 5' AGCUAGUCCUAGACCCUAAGA } 3^{\prime} \\
\text { R: } 5^{\prime} \text { UUAGGGUCUAGGACUAGCUUG 3' }\end{array}$ \\
\hline
\end{tabular}

RNAi MAX transfection reagent (Invitrogen, Carlsbad, CA, USA) according to the manufacturer's instructions. Black control that raised normally and NC-siRNA were tested. The siRNA-1, 2, 3 transfection experiments were repeated three times.

\section{Overexpression of Spp1 in Schwann cells}

$\mathrm{SCs}$ were cultured in DMEM (GIBCO, Grand Island, NY) with $100 \mathrm{IU} / \mathrm{mL}$ penicillin, $10 \%$ fetal calf serum, and $100 \mathrm{~g} / \mathrm{mL}$ streptomycin at $37{ }^{\circ} \mathrm{C}$ and $5 \% \mathrm{CO}_{2}$. The SCs were identified by examining the immunofluorescence of an antibody to the marker S100, and the final cells were found to comprise $98 \%$ SCs. The Spp1 overexpression plasmid pcDNA3.1-Spp1 was constructed as previously described [21]. A mixture of pcDNA3.1-Spp1 plasmid and X-treme GENE HP DNA Transfection Reagent (Roche, Mannheim, Germany), or X-treme GENE HP DNA Transfection Reagent and an empty vector were then transfected into SCs for $48 \mathrm{~h}$. After that, real-time quantitative (q)PCR and Western blot analyses were conducted. The pcDNA3.1-Spp1 overexpression experiments were repeated three times.

\section{Real-time quantitative PCR analysis}

Total RNA was extracted with Trizol regent, and cDNA was synthesized with a cDNA Reverse Transcription Kit (Qiagen, Valencia, CA, USA). Real-time qPCR was performed using a 7300 Real-Time PCR System according to the manufacturer's protocols. The analysis was repeated three times, and the reactions were conducted in triplicate. The comparative $\mathrm{Ct}$ method was used to analyze the cycle threshold $(\mathrm{Ct})$ values. The data were analyzed, and group differences were considered statistically significant at values of $p$ less than 0.05 .

\section{Western blot analysis}

Injured nerve samples and SCs were homogenized in protein lysis buffer containing protease inhibitors. The protein expression levels were analyzed using antibodies against anti-Spp1, AKT, phosphorylated (p)-AKT, protein kinase $\mathrm{C}$-alpha (PKC $\alpha$ ), c-Fos, extracellular signal-regulated kinase 
(ERK), and p-ERK. The Western blot images were scanned with a GS800 Densitometer Scanner (Bio-Rad, Hercules, CA, USA), and the optical density data were analyzed using PDQuest 7.2.0 software. GAPDH was used as a reference to normalize the levels of protein. The data were analyzed, and group differences were considered statistically significant at values of $p$ less than 0.05 . All injured nerve samples were analyzed in three independent experiments.

\section{Flow cytometry analysis}

The extent of SC apoptosis was measured using an Annexin V-FITC Apoptosis detection kit (Beyotime Institute of Biotechnology, China) as described by the manufacturer's instructions. SCs were washed with PBS and then collected for flow cytometry analysis. FITC-labeled annexin $\mathrm{V}(5 \mu \mathrm{L})$ in binding buffer $(195 \mu \mathrm{L})$ was incubated for $10 \mathrm{~min}$ at room temperature. The incubation was continued with $10 \mu \mathrm{L}$ of propidium iodide for $10 \mathrm{~min}$ on ice in the dark. After that, the apoptotic cells were measured by FACScan flow cytometry.

\section{Cell proliferation assay}

Cultured SCs were plated at a density of $2 \times 10^{5}$ cells $/ \mathrm{mL}$ onto $0.01 \%$ poly-L-lysine-coated plates. Cell proliferation was assayed at 2 days after cell transfection. EdU $(50 \mu \mathrm{M})$ was added to the cell culture and incubated for $2 \mathrm{~h}$. The SCs were then fixed with $4 \%$ formaldehyde for $30 \mathrm{~min}$. After SC labeling, a Cell-Light EdU DNA Cell Proliferation Kit (Ribobio, China) was used to analyze cell proliferation according to the manufacturer's protocol. Cell proliferation was expressed as the ratio of EdU-positive cells, which was defined by images of randomly selected fields obtained

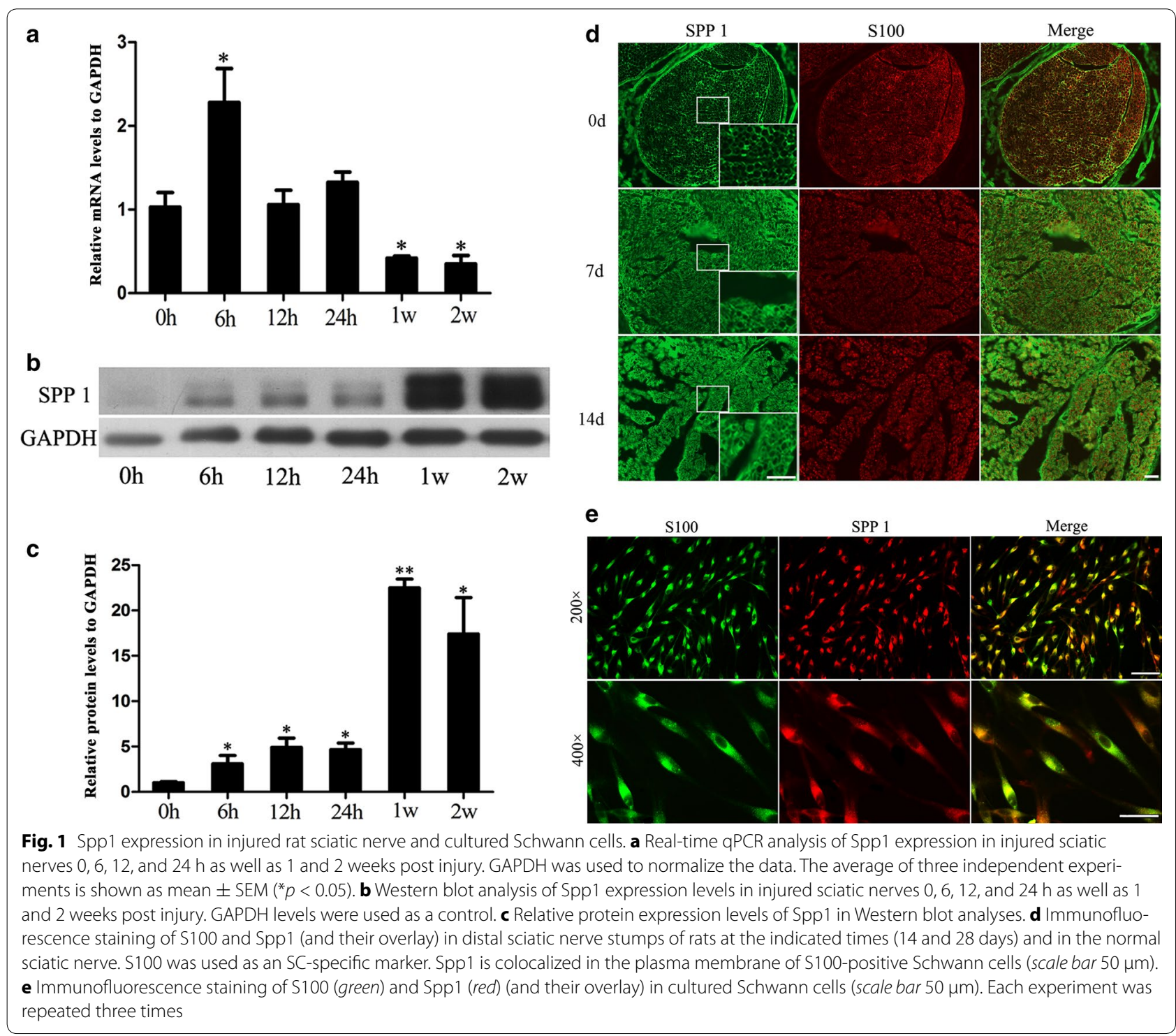



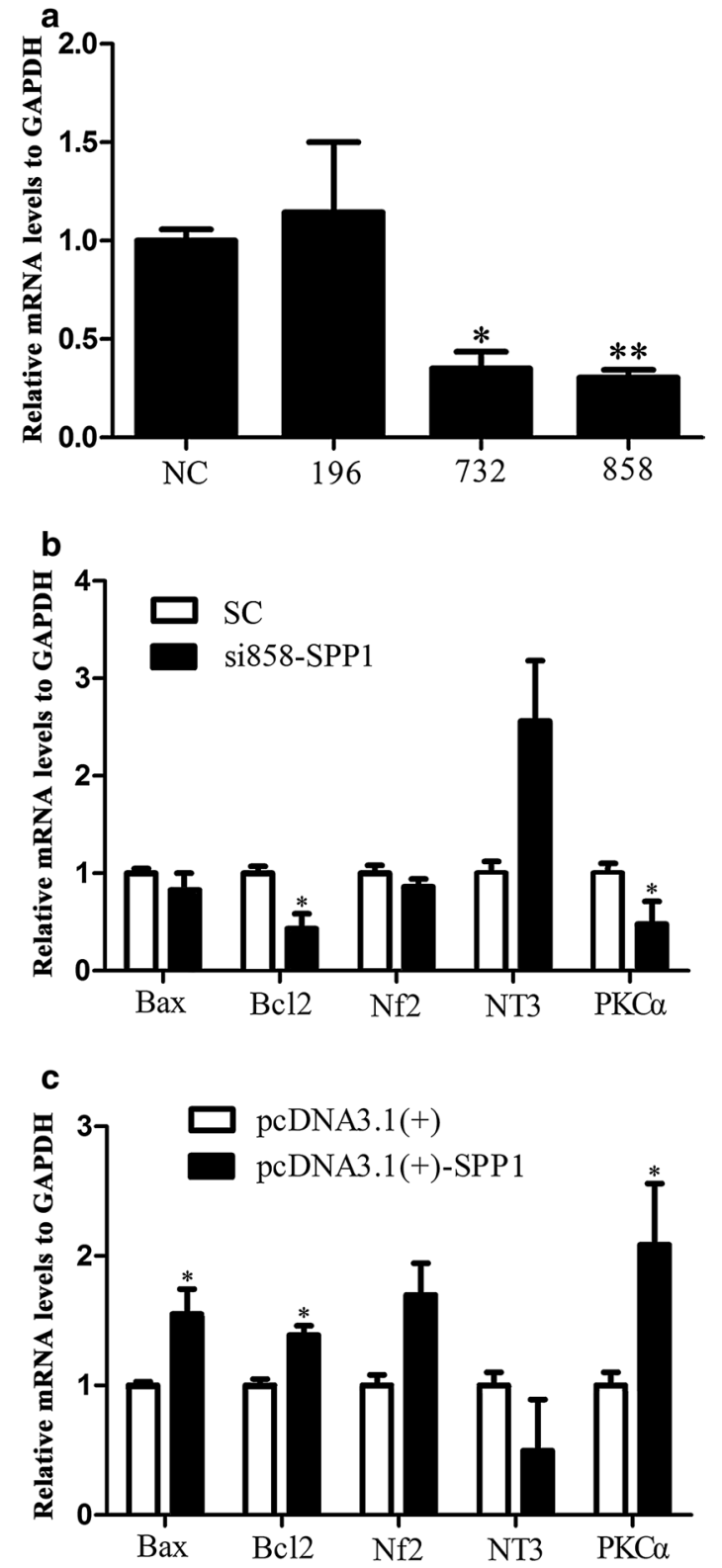

Fig. 2 Cytokine expression in SC Spp1 knockdown and overexpression. a Spp1 siRNA-1, 2, 3 transfection efficiency assay $\left(^{*} p<0.05\right)$. b Real-time qPCR analysis of bax, bcl2, nf2, NT3, and PKCa mRNA expression levels after Spp1 siRNA-858 transfection of SCs for 2 days. GAPDH was used to normalize values to the negative control $\left({ }^{*} p<0.05\right)$. The average of three independent experiments is shown as mean $\pm \mathrm{SEM}$. c Real-time qPCR analysis of bax, bcl2, nf2, NT3, and PKCa mRNA expression levels after pcDNA3.1-Spp1 plasmid transfection of SCs for 2 days, using GAPDH to normalize data to the negative control $\left({ }^{*} p<0.05\right)$. The average of three independent experiments is shown as mean \pm SEM

on a DMR fluorescence microscope (Leica Microsystems, Bensheim, Germany). The cell proliferation assays were performed three times using triplicate wells.

\section{Cell migration assay}

Transwell chambers $(6.5 \mathrm{~mm})$ with 8 - $\mu$ m pores were used to examine SC migration as described previously [21]. SCs $\left(10^{6}\right.$ cells $\left./ \mathrm{mL}\right)$ resuspended in $100 \mu \mathrm{L}$ of DMEM were transferred to the top chamber and allowed to migrate in $5 \% \mathrm{CO}_{2}$ into the lower chamber before the addition of $600 \mu \mathrm{L}$ complete medium. Cells adhering to the bottom surface of each membrane were stained with $0.1 \%$ crystal violet, imaged, and counted using a DMR inverted microscope (Leica Microsystems, Bensheim, Germany). The cell migration assays were conducted three times using triplicate wells.

\section{Immunohistochemistry}

The distal sciatic nerve samples were fixed with $4 \%$ paraformaldehyde and dehydrated in 30\% sucrose solution. Sections were cut using a cryostat to a thickness of $12 \mu \mathrm{m}$ and mounted onto slides. The sections were rinsed in PBS, permeabilized in $0.3 \%$ Triton $\mathrm{X}-100,5 \%$ goat serum, and $1 \%$ BSA in PBS, and then stained. The sections were incubated with mouse monoclonal anti-S100 (1:400, Sigma) and Spp1 (1:50, Santa Cruz) antibodies at $4{ }^{\circ} \mathrm{C}$ for $12 \mathrm{~h}$, and then incubated with goat anti-mouse or goat anti-rabbit IgG Cy3 (1:400, Sigma) and IgG Alexa Fluor 488 (1:400, Invitrogen) at room temperature for $2 \mathrm{~h}$. The sections were counterstained with Hoechst 33342 for $5 \mathrm{~min}$. All samples were observed under a fluorescence microscope. Images were acquired using a laser microscope (FV10i-oil, Tokyo, Japan).

\section{In vivo assay}

The sciatic nerve of adult male Sprague-Dawley rats was exposed through an incision on the left hind limb and cut to create a $1-\mathrm{cm}$ gap. A silicone tube (i.d., $1.0 \mathrm{~mm}$ ) was implanted to bridge the nerve gap. The rats were randomly divided into two groups $(n=3$ each): Spp1 siRNA injected into the tube after the nerve gap bridge for the experimental group, and a control group. At 7 and 14 days after surgery, the rats were killed, and the silicone tubes together with the regenerated nerves were collected. Real-time PCR and Western blot analyses were conducted. The nerve samples (7 and 14 days) were analyzed in three independent experiments.

\section{Statistical analysis}

Statistical analyses were performed using SPSS 15.0 for windows (SPSS, Chicago, IL, USA). Group differences were analyzed by one-way analysis of variance and Scheffés post hoc test when appropriate. Student's $t$ test was used for comparisons between two groups. Values of $p$ less than 0.05 were considered statistically significant. All data are expressed as mean $\pm \mathrm{SD}$. 


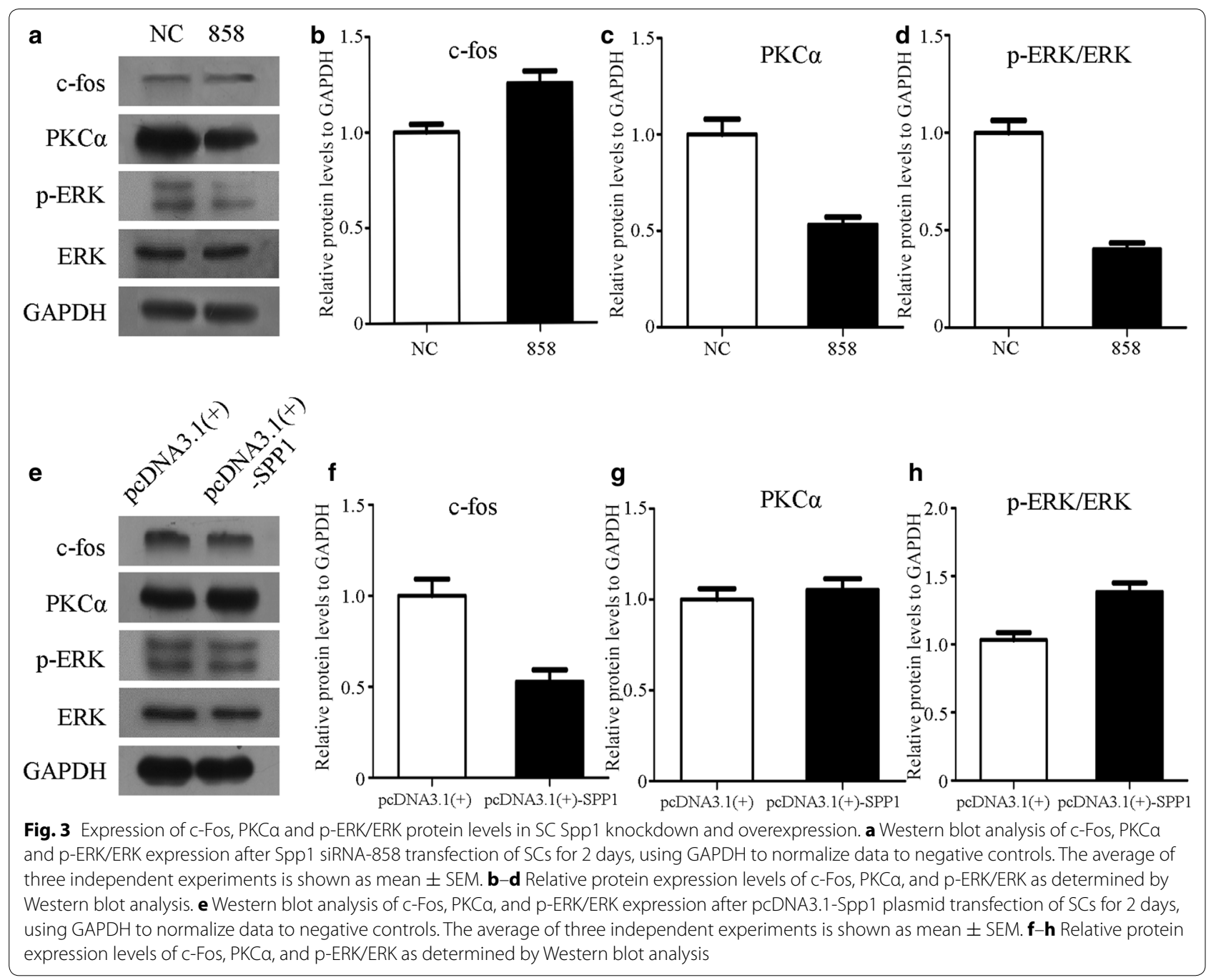

\section{Results}

Spp1 is expressed in injured sciatic nerves and in Schwann cells

We used real-time qPCR and Western blot analyses to determine the expression of Spp1 0, 6, 12, and 24 h as well as 1 and 2 weeks after sciatic nerve injury. The realtime qPRC results indicated that Spp1 mRNA expression was increased from 6 to 24 h after injury and then decreased. Spp1 mRNA level was significantly higher at $6 \mathrm{~h}$ after injury. Our western blot assay results indicated that Spp1 protein expression was increased from $6 \mathrm{~h}$ to 2 weeks. Spp1 protein level was significant higher at $1 \mathrm{w}$ after injury. GAPDH levels were used as a control (Fig. 1a-c). We used immunohistochemistry to visualize the location of Spp1 and S100 at 0, 14, 28 days after sciatic nerve injury and in cultured Schwann cells. The Schwann cells were immunostained with anti-S100, which is a specific Schwann cell marker. The results of our immunostaining assay demonstrated that Spp1 and S100 were colocalized in Schwann cells (Fig. 1d), indicating that Spp1 is expressed in the Schwann cells of the sciatic nerve. We also examined the expression of Spp1 in cultured Schwann cells. The data indicated that Spp1 was also present in cultured Schwann cells $(p<0.05$, vs. day 0 ; Fig. 1e). All data were analyzed using one-way analysis of variance and Scheffé's post hoc tests $\left({ }^{*} p<0.05\right)$.

\section{Spp1 knockdown and overexpression in transfected SCs alters mRNA expression levels}

We synthesized three specific Spp1 siRNAs-siRNA1, siRNA-2 and siRNA-3-and all three were found to reduce Spp1 mRNA expression levels. The interference transfection efficiencies of two of the siRNA fragments were at least $80 \%$ (Fig. 2a); thus, we selected the most efficient one, siRNA-3 (siRNA-858), for the following experiment. To investigate the potential functions of 
a

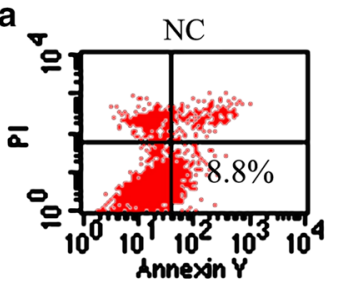

b.
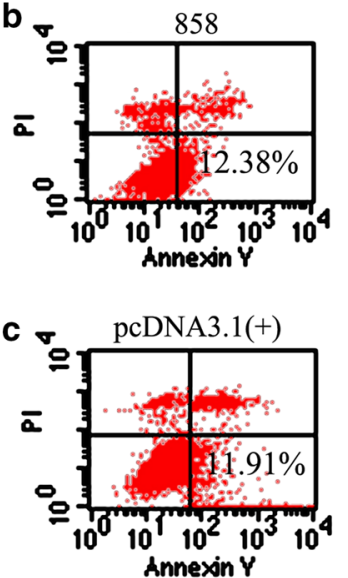

d

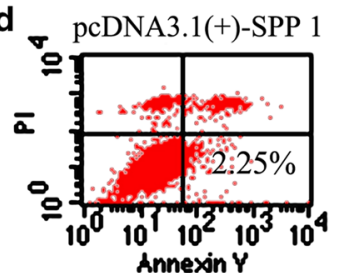

Quad Events \% Galled \% Total XMean YMean

$\begin{array}{rrrrrr}\text { UL } & 474 & 8.41 & 4.74 & 18.82 & 228.83 \\ \text { UR } & 204 & 3.62 & 2.04 & 185.90 & 288.11 \\ \text { LL } & 4465 & 79.18 & 44.65 & 19.10 & 5.33\end{array}$

$\begin{array}{llllll}\text { LR } & 496 & 8.80 & 4.96 & 54.90 & 10.24\end{array}$

\begin{tabular}{rrrrrrr} 
Quad & Events & \& Gated & X Total & X Mean & Y Mean \\
\hline UL & 316 & 5.60 & 3.16 & 16.98 & 191.13 \\
UR & 267 & 4.74 & 2.67 & 200.19 & 294.81 \\
LL & 4357 & 77.28 & 43.57 & 20.57 & 5.13 \\
LR & 698 & 12.38 & 6.98 & 53.72 & 9.15
\end{tabular}

Quad Events 8 Gated 8 Total XMean YMean

$\begin{array}{rrrrrr}\text { UL } & 286 & 3.32 & 2.86 & 24.60 & 456.37 \\ \text { UR } & 492 & 5.71 & 4.92 & 239.59 & 510.40 \\ \text { LL } & 6818 & 79.07 & 68.18 & 24.45 & 14.38 \\ \text { LR } & 1027 & 11.91 & 10.27 & 887.98 & 6.93\end{array}$

\begin{tabular}{rrrrrrr} 
Quad & Events & X Gated & X Tota & X Mean & Y Mean \\
\hline UL & 276 & 3.13 & 2.76 & 26.03 & 517.88 \\
UR & 313 & 3.55 & 3.13 & 340.49 & 521.88 \\
LL & 8018 & 91.06 & 80.18 & 20.70 & 12.31 \\
LR & 198 & 2.25 & 1.98 & 224.17 & 20.37
\end{tabular}
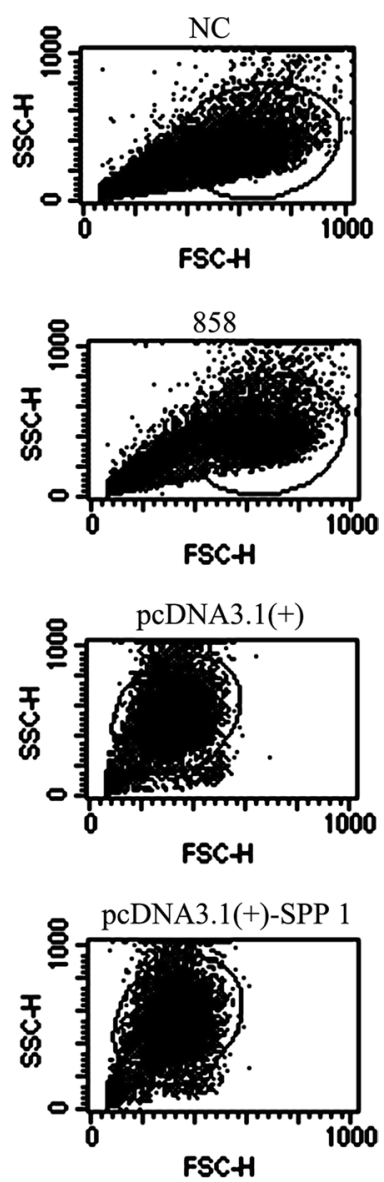

e

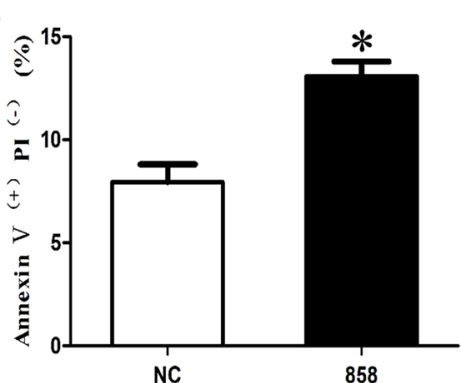

f

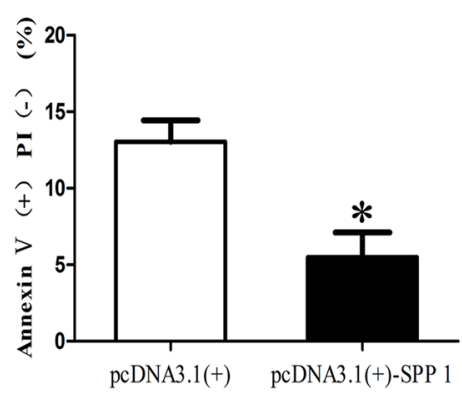

Fig. 4 Spp1 knockdown and overexpression affect SC apoptosis. a, b Silencing Spp1 by transfection with Spp1 siRNA significantly induces apoptosis of SCs compared with that in negative controls (NC). c, d Overexpression of Spp1 in SCs by transfection with the pcDNA3.1-Spp1 plasmid significantly inhibits SC apoptosis compared with that in NCs. e Relative numbers of SCs with the Spp1 knockdown. f Relative numbers of SCs overexpressing Spp1. The average of three independent experiments is shown as mean $\pm \operatorname{SEM}\left({ }^{*} p<0.05\right)$

Spp1 in SCs, including on cytokine release, we analyzed the mRNA levels of the pro-apoptotic factors B-cell lymphoma 2 (Bcl2) and Bcl-2-associated $\mathrm{X}$ protein (Bax) as well as neurofibromin 2 (Nf2), neurotrophin 3 (NT3) and PKC $\alpha$ after Spp1 knockdown and overexpression in transfected SCs. Our real-time qPCR results showed that bax, bcl2, Nf2 and PKC $\alpha$ mRNA expression levels were downregulated in SCs with Spp1 knocked down and upregulated by Spp1 overexpression in SCs. By contrast, the expression of NT3 mRNA was upregulated by Spp1 knockdown and downregulated by Spp1 overexpression in SCs (Fig. 2b, c) (" $p<0.05)$. These data indicated that the differential expression of Spp1 alters mRNA expression levels in SCs. 

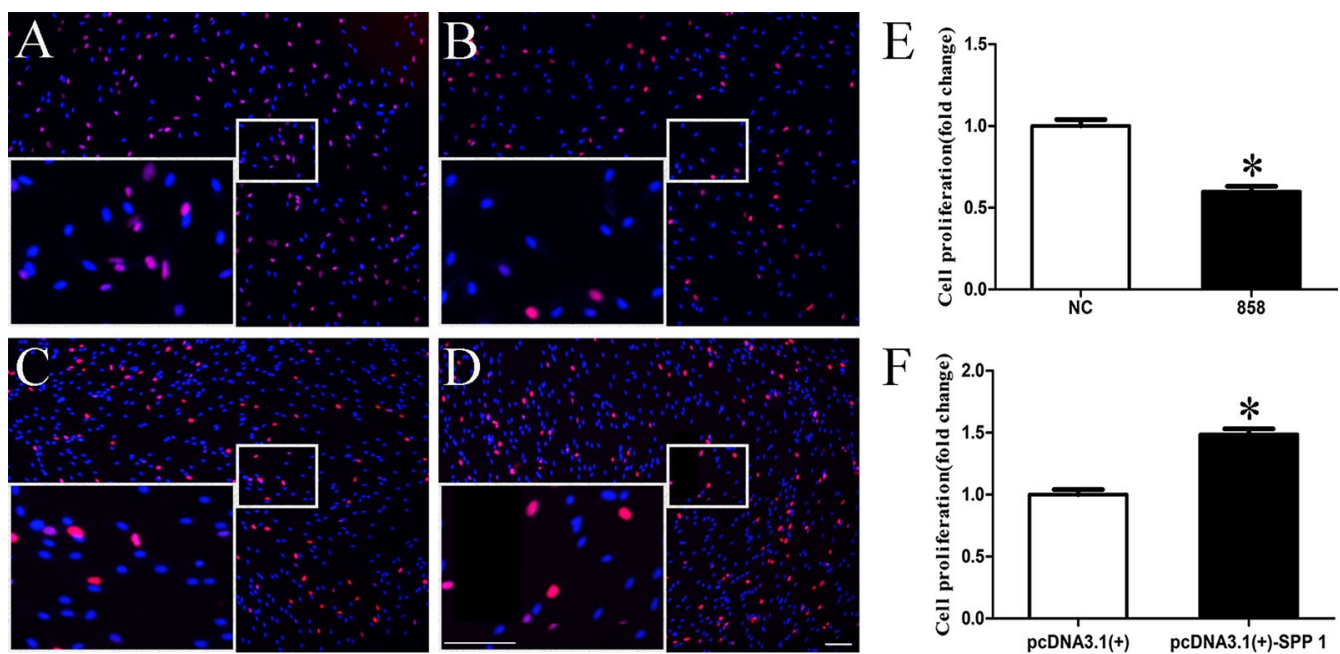

Fig. 5 Spp 1 knockdown and overexpression affect SCs proliferation. a, b Silencing of Spp 1 by transfection with Spp 1 siRNA significantly inhibits SC proliferation compared with that in negative controls (NCs). c, d Overexpression of Spp1 by transfection with the pcDNA3.1-Spp1 plasmid significantly induces SC proliferation compared with that in NCs. e Relative numbers of SCs with the Spp1 knockdown. f Relative numbers of SCs overexpressing Spp1 (scale bar $100 \mu \mathrm{m})$. The average of three independent experiments is shown as mean $\pm \operatorname{SEM}\left({ }^{*} p<0.05\right)$
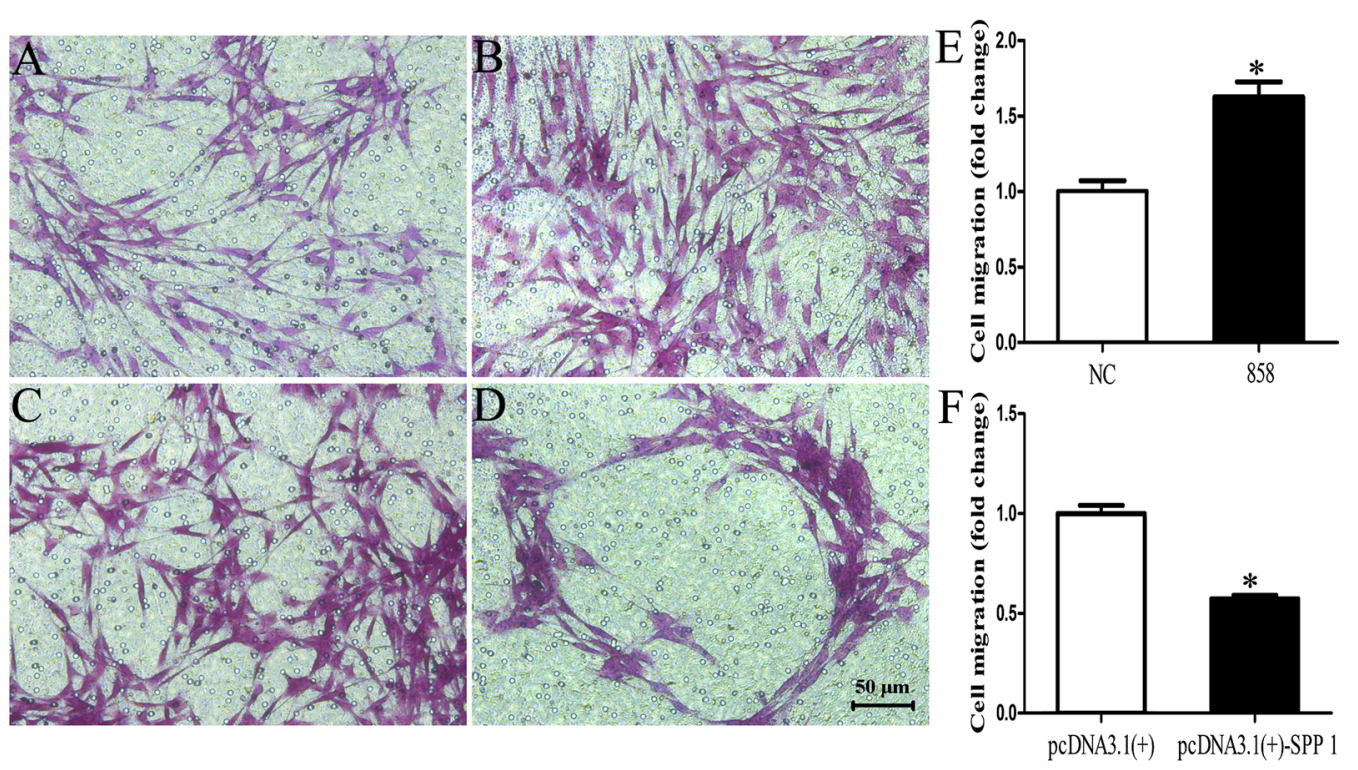

Fig. 6 Spp1 knockdown and overexpression affect SCs migration. a, b Silencing of Spp1 by transfection with Spp1 siRNA in SCs significantly induces SC migration compared with that in negative controls (NCS). c, d Overexpression of Spp 1 by transfection with the pcDNA3.1-Spp1 plasmid significantly inhibits SC migration compared with that in NCs. e Relative numbers of SCs with the Spp1 knockdown. f Relative numbers of SCS overexpressing Spp1. The average of three independent experiments is shown as mean \pm SEM $\left({ }^{*} p<0.05\right)$

\section{Spp1 affects c-Fos, PKCa, and ERK signaling pathways in vitro}

As shown above, altered Spp1 expression resulted in changes in mRNA expression levels that could affect cytokines. We next examined whether Spp1 affected signaling pathways in vitro using cultured SCs. Protein expression levels of c-Fos, PKC $\alpha$ and p-ERK/ERK were compared with those in negative control SCs using Western blot analysis. We found that protein levels of c-Fos, PKC $\alpha$ and $p$-ERK/ERK were significantly changed after transfection of Spp1 siRNA and the pcDNA3.1-Spp1 plasmid (" $p<0.05$ ), indicating that $\mathrm{c}$-Fos, PKC $\alpha$ and p-ERK/ERK signaling pathways could be activated by Spp1 (Fig. 3). Thus, Spp1 may play roles in regulating 


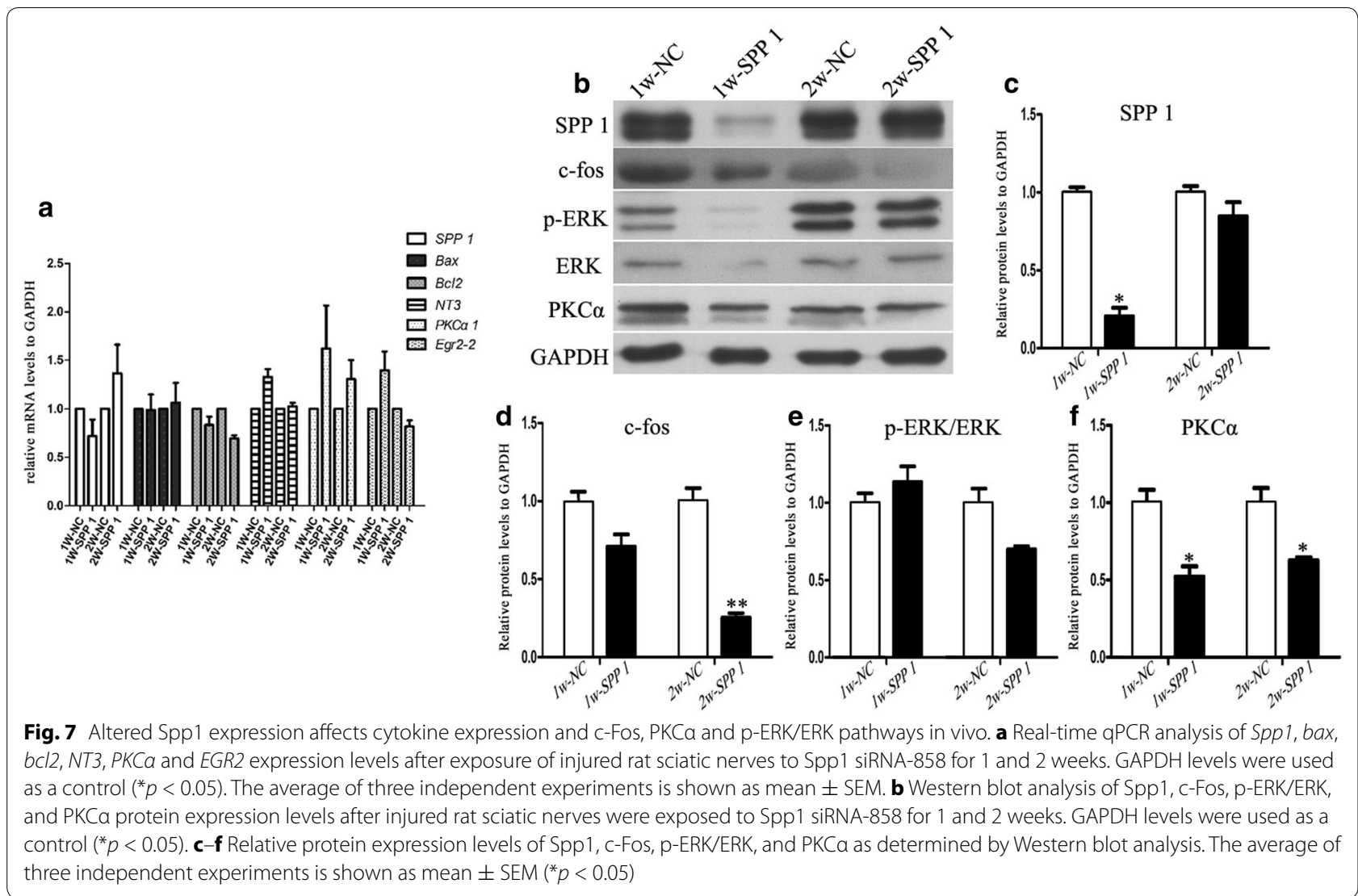

c-Fos, PKC $\alpha$, and p-ERK/ERK signaling pathways in cultured SCs in vitro.

\section{Spp1 affects SC apoptosis, proliferation, and migration in vitro}

To determine the function of Spp1 in SCs, primary SCs were transfected with Spp1 siRNA, pcDNA3.1-Spp1 plasmid, or negative control vector, and the effects of these transfections on cell apoptosis, proliferation, and migration of SCs were examined in vitro. Compared with that in negative controls, the apoptosis rate of SCs transfected with Spp1 siRNA was increased (1.5-fold) and that of SCs transfected with pcDNA3.1-Spp1 was decreased (2.0fold; Fig. 4) (" $p<0.05)$, suggesting that silencing Spp1 induced SC apoptosis, whereas enhancing Spp1 expression reduced SC apoptosis. The results of our EdU-based proliferation assay indicated that compared with the proliferation rate of SCs in negative controls, that of SCs transfected with Spp1 siRNA was decreased (1.8-fold), whereas that of SCs transfected with the pcDNA3.1Spp1 plasmid was increased (1.5-fold; Fig. 5) (" $p<0.05$ ). We also found that the migration rate of SCs transfected with Spp1 siRNA was increased (1.6-fold), whereas that of SCs transfected with the pcDNA3.1-Spp1 plasmid was decreased (1.8-fold) as compared with that of the negative control (Fig. 6) $(" p<0.05)$. These results indicated that the expression of Spp1 affects SC apoptosis, proliferation, and migration in vitro.

\section{Altered Spp1 expression affects sciatic nerve injury was been assed in vivo}

To determine the effect of Spp1 on WD after rat sciatic nerve injury in vivo, we examined the functions of Spp1 on rat sciatic nerve repair and regeneration 1 and 2 weeks after injury. After exposing the injured sciatic nerves to either Spp1 siRNA or the negative control vector for 1 or 2 weeks, we conducted real-time qPCR and Western blot analyses. Our results indicated that altered Spp1 expression affected the mRNA and protein expression levels of the cytokines $\mathrm{Bax}, \mathrm{Bcl} 2$, NT3, and early growth response 2 (EGR2) as well as of PKC $\alpha$ and differentially regulated the c-Fos, PKC $\alpha$ and p-ERK/ERK signaling pathways (Fig. 7) (" $p<0.05)$. These results were consistent with the functions of Spp1 observed in vitro. 


\section{Discussion}

Peripheral nerve injury and repair is a result of reactivated regeneration mechanisms in combination with newly activated injury-dependent reactions. Our data indicated that gene and protein expression changes after sciatic nerve injury appeared to provide separate signals that were characterized by a high degree of overlapping genes [22-25]. These signals are thought to recruit neutrophils and may amplify proinflammatory cytokine responses via the phosphatidylinositol 3-kinase/nuclear factor-kappa $B$ pathway to activate the processes of nerve injury, repair, and regeneration [21, 26-33].

In the present study, we determined the effect of Spp1 on nerve repair and rejuvenation after sciatic nerve injury in vitro and vivo. Spp1 has been described as a component of the inflammatory environment of dystrophic and injured tissues [17]. Different cell types may differ in their regulatory mechanisms of the Spp1 gene. Although Spp1 is also important for the migration of neutrophils in vitro [34-42], regulation of the Spp1 gene is incompletely understood. Our results indicated that Spp1 mediates cell activation and cytokine production after sciatic nerve injury. In addition, Spp1 may act as an important anti-apoptotic factor and may prevent non-programmed cell death in inflammatory colitis. Spp1 was previously shown to act as a macrophage chemotactic factor and play an important role in mast cell migration [17]. Here, we reported the functions of Spp1 in the injured sciatic nerve during WD. Stimulation of Spp1 expression resulted in cytokine expression changes and may regulate c-Fos, $\mathrm{PKC} \alpha$ and $\mathrm{p}$-ERK/ERK pathways in vitro. Altered Spp1 expression was also shown to affect SC proliferation, migration, and apoptosis. We also verified these data in vivo. Further studies will be necessary to identify the key regulatory factors, how they regulate signaling pathways in vivo, and their functions during WD after peripheral nerve injury.

\section{Conclusions}

Spp1 is differentially expressed during WD after rat sciatic nerve injury. In vitro and vivo analyses revealed that Spp1 is a key regulatory factor that affects nerve degeneration and regeneration through c-Fos, $\mathrm{PKC} \alpha$, and p-ERK/ERK pathways after rat sciatic nerve injury. We concluded that Spp1 plays important roles in peripheral nerve injury, repair, and regeneration.

\footnotetext{
Abbreviations

Bax: Bcl-associated X protein; bcl-2: B-cell lymphoma-2; bFGF: basic fiberoblast growth factor; CNS: central nervous system; ERK: extracelluler regulated protein kinase; GAPDH: glyceraldehyde-3-phosphate dehydrogenase; JaK: Janus kinase; JNK: c-Jun NH2-terminal kinase; NF2: neurofibromin 2; NT3: neurotrophin 3; PKC: protein kinase C; PNS: peripheral nervous system; SC: Schwann
}

cells; siRNA: small interfering RNA; Spp1: secreted phosphoprotein 1;TLR: toll like receptor.

\section{Authors' contributions}

$\mathrm{XL}$ and YS performed the experiments; HL conducted the animal studies; RC performed analytical studies; $M L$ and $Y Y$ were responsible for gene expression analysis; $Y L$ and $Y Z$ performed immunohistochemical experiments; $X L$ analysed functional and biochemical data; SC and DY planned the study; DY wrote the manuscript. All authors read and approved the final manuscript.

\begin{abstract}
Author details
${ }^{1}$ China-Japan Union Hospital of Jilin University, 126 Xiantai Road, Changchun 130033, Jilin, People's Republic of China. ${ }^{2}$ School of Life Sciences, Jiangsu Key Laboratory of Neuroregeneration, Co-Innovation Center of Neuroregeneration, Nantong University, 19 Qixiu Road, Nnatong 226001, Jiangsu, People's Republic of China. ${ }^{3}$ Affiliated Hospital of Nantong University, 20 Xisi Road, Nantong 226001, Jiangsu, People's Republic of China.
\end{abstract}

\section{Acknowledgements}

We greatly appreciate the editorial assistance of lan Haigler.

\section{Competing interests}

The authors declare that they have no competing interests.

Availability of data and materials

Raw data can be provided upon request.

\section{Ethical approval and consent to participate}

All animal tests were conducted in accordance with the US National Institutes of Health's Guide for the Care and Use of Laboratory Animals and by the Key Laboratory of Neuroregeneration Guidelines for the Care and Use of Laboratory Animals. The Institutional Animal Care and Use Committee of Nantong University approved all protocols used in this study.

\section{Fundings}

This research was supported by Grants from: the National Natural Science Foundation of China (Grant Nos. 81370982; 81471259; 81671220; Key Program, Grant No. 81130080); Scientific Research Foundation for Returned Scholars, Ministry of Education of China; Science and Technology Development Project of Jilin Province (Grant No. 20150311069 YY); A Project Funded by the Priority Academic Program Development of Jiangsu Higher Education Institutions, PAPD.

Received: 8 November 2016 Accepted: 24 February 2017 Published online: 07 March 2017

\section{References}

1. Ghergherehchi $\mathrm{CL}$, et al. Effects of extracellular calcium and surgical techniques on restoration of axonal continuity by polyethylene glycol fusion following complete cut or crush severance of rat sciatic nerves. J Neurosci Res. 2016;94:231-45.

2. Siqueira $M B$, et al. Role of IL-10 in resolution of inflammation and functional recovery after peripheral nerve injury. J Neurosci. 2015;35:16431-42.

3. Chen P, Piao X, Bonaldo P. Role of macrophages in Wallerian degeneration and axonal regeneration after peripheral nerve injury. Acta Neuropathol. 2015;130:605-18

4. Wang $L$, et al. Role of Schwann cells in the regeneration of penile and peripheral nerves. Asian J Androl. 2015;17:776-822

5. Gong $L$, et al. The effects of claudin 14 during early Wallerian degeneration after sciatic nerve injury. Neural Regen Res. 2014;9:2151-8.

6. Noble J, et al. Analysis of upper and lower extremity peripheral nerve injuries in a population of patients with multiple injuries. J Trauma. 1998:45:116-22.

7. $\mathrm{Yu} \mathrm{H}$, et al. ERK1/2 and AKT are vital factors in regulation of the migration of rat Schwann cells. J Vet Med Sci. 2015:77:427-32.

8. Li M, et al. TGF- $\beta 1$ is critical for Wallerian degeneration after rat sciatic nerve injury. Neuroscience. 2015;22(284):759-67. 
9. Li M, et al. Protein expression profiling during Wallerian degeneration after rat sciatic nerve injury. Muscle Nerve. 2014;50:73-8.

10. Yao D, et al. Expression changes and bioinformatic analysis of Wallerian degeneration after sciatic nerve injury in rat. Neurosci Bull. 2013;29:321-32.

11. Li M, et al. Signal flow and pathways in response to early Wallerian degeneration after rat sciatic nerve injury. Neurosci Lett. 2013;536:56-63.

12. Boivin A, et al. Toll-like receptor signaling is critical for Wallerian degeneration and functional recovery after peripheral nerve injury. J Neurosci. 2007;27:12565-76.

13. Gaudet AD, Popovich PG, Ramer MS. Wallerian degeneration: gaining perspective on inflammatory events after peripheral nerve injury. J Neuroinflammation. 2011:8:110

14. Stoll $G$, Jander $S$. The role of microglia and macrophages in the pathophysiology of the CNS. Prog Neurobiol. 1999;58:233-47.

15. Vargas ME, Barres BA. Why is Wallerian degeneration in the CNS so slow? Annu Rev Neurosci. 2007;30:153-79.

16. Jessen KR, Mirsky R. Schwann cells and their precursors emerge as major regulators of nerve development. Trends Neurosci. 1999;22:402-10.

17. Pegoraro E, et al. SPP1 genotype is a determinant of disease severity in Duchenne muscular dystrophy. Neurology. 2011;76:219-26.

18. Yuan $\mathrm{H}$, et al. The protective effects of resveratrol on Schwann cells with toxicity induced by ethanol in vitro. Neurochem Int. 2013;63:146-53.

19. Liu N, et al. microRNA-206 promotes skeletal muscle regeneration and delays progression of Duchenne muscular dystrophy in mice. J Clin Invest. 2012;122:2054-65.

20. Mantuano E, Inoue G, Li X, Takahashi K, Gaultier A, Gonias SL, Campana WM. The hemopexin domain of matrix metalloproteinase- 9 activates cell signaling and promotes migration of schwann cells by binding to low-density lipoprotein receptor-related protein. J Neurosci. 2008;28(45):11571-82

21. Wang $Y$, Hu C, Dong R, et al. Platelet-derived growth factor-D promotes ovarian cancer invasion by regulating matrix metalloproteinases 2 and 9 . Asian Pac J Cancer Prev. 2011;12:3367-70.

22. Love FM, Thompson WJ. Schwann cells proliferate at rat neuromuscular junctions during development and regeneration. J Neurosci. 1998;18:9376-85.

23. Arthur-Farraj PJ, et al. c-Jun reprograms Schwann cells of injured nerves to generate a repair cell essential for regeneration. Neuron. 2012;75:633-47.

24. Chen Z, et al. Skin-derived precursors as a source of progenitors for cutaneous nerve regeneration. Stem Cells. 2012;30:2261-70.

25. Kingham PJ, Terenghi G. Bioengineered nerve regeneration and muscle reinnervation. J Anat. 2006;209:511-26.

26. Navarro X, Vivo M, Valero-Cabre A. Neural plasticity after peripheral nerve injury and regeneration. Prog Neurobiol. 2007;82:163-201.
27. Peltonen S, Alanne M, Peltonen J. Barriers of the peripheral nerve. Tissue Barriers. 2013;3(e24956):1-6.

28. Raivich $\mathrm{G}$, et al. The AP-1 transcription factor $\mathrm{c}$-Jun is required for efficient axonal regeneration. Neuron. 2004;43:57-67.

29. Burnett MG, Zager EL. Pathophysiology of peripheral nerve injury: a brief review. Neurosurg Focus. 2004;16:E1.

30. Liu HM, Yang LH, Yang YJ. Schwann cell properties: 3. C-fos expression, bFGF production, phagocytosis and proliferation during Wallerian degeneration. J Neuropathol Exp Neurol. 1995:54:487-96.

31. Zarbakhsh S, et al. The effects of schwann and bone marrow stromal stem cells on sciatic nerve injury in rat: a comparison of functional recovery. Cell J. 2012;14:39-46.

32. Mirsky R, Jessen KR. Schwann cell development, differentiation and myelination. Curr Opin Neurobiol. 1996;6:89-96.

33. Parkinson DB, et al. Transforming growth factor beta (TGF-beta) mediates Schwann cell death in vitro and in vivo: examination of c-Jun activation, interactions with survival signals, and the relationship of TGFbeta-mediated death to Schwann cell differentiation. J Neurosci. 2001;21:8572-85.

34. Song $L$, et al. Dab2 attenuates brain injury in APP/PS1 mice via targeting transforming growth factor-beta/SMAD signaling. Neural Regen Res. 2014;9:41-50

35. Taskinen HS, et al. Distinct expression of TGF-beta1 mRNA in the endoand epineurium after nerve injury. J Neurotrauma. 2004;21:969-75.

36. Echeverry S, Wu Y, Zhang J. Selectively reducing cytokine/chemokine expressing macrophages in injured nerves impairs the development of neuropathic pain. Exp Neurol. 2013;240:205-18.

37. Nie $X$, et al. Axonal regeneration and remyelination evaluation of chitosan/gelatin-based nerve guide combined with transforming growth factor-beta1 and Schwann cells. Cell Biochem Biophys. 2014;68:163-72.

38. Hsu M, Stevenson FF. Wallerian degeneration and recovery of motor nerves after multiple focused cold therapies. Muscle Nerve. 2015;51:268-75.

39. Sulaiman W, Dreesen TD. Effect of local application of transforming growth factor-beta at the nerve repair site following chronic axotomy and denervation on the expression of regeneration-associated genes. J Neurosurg. 2014;121:859-74.

40. DeFrancesco-Lisowitz A, Lindborg JA, Niemi JP, Zigmond RE. The neuroimmunology of degeneration and regeneration in the peripheral nervous system. Neuroscience. 2015;27:174-203.

41. Park BS, et al. Hydrogen sulfide is essential for Schwann cell responses to peripheral nerve injury. J Neurochem. 2015;132:230-42.

42. Bastien D, Lacroix S. Cytokine pathways regulating glial and leukocyte function after spinal cord and peripheral nerve injury. Exp Neurol. 2014;258:62-77.

\section{Submit your next manuscript to BioMed Central and we will help you at every step:}

- We accept pre-submission inquiries

- Our selector tool helps you to find the most relevant journal

- We provide round the clock customer support

- Convenient online submission

- Thorough peer review

- Inclusion in PubMed and all major indexing services

- Maximum visibility for your research

Submit your manuscript at www.biomedcentral.com/submit
BioMed Central 